\title{
Behavioural phenotype of Cornelia de Lange syndrome: case-control study
}

Chris Oliver, Kate Arron, Jenny Sloneem and Scott Hall

\section{Background}

Cornelia de Lange syndrome is associated with abnormalities on chromosomes 5, 10 and $\mathrm{X}$.

\section{Aims \\ To delineate the behavioural phenotype of Cornelia de Lange syndrome with specific reference to autistic-spectrum disorder. \\ Method \\ A total of 54 individuals with Cornelia de Lange syndrome (mean age 13.88 years; s.d. $=8.58$ ) and 46 comparable individuals with intellectual disability (mean age 13.74 years; s.d.=7.99) were assessed on measures of autistic-spectrum disorder, and adaptive, compulsive and disordered behaviour.}

\section{Results}

There was no difference between the groups in global behaviour disorder. Severe autism was significantly more prevalent in the syndrome group (32.1\%) than the comparison group (7.1\%). In addition, the syndrome group also evidenced significantly higher levels of compulsive behaviour.

\section{Conclusions}

These data suggest that autistic-spectrum disorder is part of the behavioural phenotype of Cornelia de Lange syndrome and that compulsive behaviours are evident. Future research should investigate this behavioural phenotype using contemporary diagnostic algorithms for autism with detailed examination of the phenomenology of compulsive behaviours.

\section{Declaration of interest}

None. Funding detailed in Acknowledgements.
Cornelia de Lange syndrome is associated with abnormalities on chromosomes 5, 10 and $\mathrm{X}^{1-4}$ The physical phenotype includes small stature, limb abnormalities, facial dysmorphology and gastrointestinal and kidney disorders. ${ }^{5}$ Degree of intellectual disability ranges from mild to profound, ${ }^{6,7}$ expressive communication is relatively poor ${ }^{8}$ and self-injurious behaviour is prominent. ${ }^{9,10}$ There is evidence for phenotypic differences between genotypes and a distinction is drawn between 'classic' and 'mild' presentations. ${ }^{11}$

'Autistic-like' behaviours are reported ${ }^{12}$ and estimates of autistic-spectrum disorder vary from $53 \%{ }^{6}$ to $89 \% .^{13}$ However, as few studies have employed matched comparison groups the interpretation of these figures is problematic. Reports of autistic-like behaviour in Cornelia de Lange syndrome note repetitive and ritualistic behaviours. Hyman et al ${ }^{14}$ reported high levels of compulsive-like behaviours with $87.5 \%$ showing at least one form. This high prevalence warrants further investigation by examining whether these behaviours are independent of autisticlike impairments.

In this study we examine communication, compulsive and autistic-like behaviour in Cornelia de Lange syndrome. We predict that these characteristics will be more prominent in people with this syndrome ('syndrome group') than in a comparison group. We also investigate the relationship between compulsive and autistic-like behaviour. We employ psychometrically robust methods of assessment and a mixed-aetiology intellectual disability group matched on key characteristics (age, gender, intellectual ability and mobility) for comparison.

\section{Method}

\section{Participants}

Families were contacted via the Cornelia de Lange Syndrome Foundation support group in the UK. Selection for inclusion was based on age (over 2 years) and location (living near one of five bases). A total of 59 participants with Cornelia de Lange syndrome were identified and all agreed to participate. Since genetic diagnosis was not available at the time of the study, clinical diagnosis was employed. Overall, 51 participants had received a diagnosis of Cornelia de Lange syndrome from a medical professional or professional specialising in the syndrome. The 8 participants with a diagnosis from another source and 5 participants for whom a diagnosis was queried were either confirmed or rejected by a clinical geneticist, eliminating 5 participants. Thus, a sample of 54 individuals with Cornelia de Lange syndrome was formed. All parents or carers gave consent to participate in the study.

To identify a comparison group, two methods were adopted. Teachers and key workers of participants with the syndrome identified up to 2 individuals who were similar to the index participant in terms of age, gender, mobility and ability $(n=5)$. Second, schools and day centres already visited distributed 876 information packs to parents and carers. Altogether, 153 $(17.5 \%)$ returned consent forms and questionnaires (including the Wessex Scale, ${ }^{15}$ see Measures). Individuals were matched to participants with Cornelia de Lange syndrome in terms of age ( \pm 2 years), gender, wheelchair use ('never', 'sometimes' or 'often') and self-help skills as determined by Wessex scores. A total of 46 comparison participants were identified.

Information on the Wessex self-help scale, mobility, age and gender are presented in Table 1. No specific diagnosis was made in 25 individuals in the comparison group; 21 had syndrome diagnoses including: Down syndrome $(n=8)$; autism $(n=3)$; cerebral palsy $(n=2)$; congenital rubella $(n=1)$; fragile-X syndrome $(n=1)$; Ito syndrome $(n=1)$; Landau-Kleffner syndrome $(n=1)$; Miller-Dieker syndrome $(n=1)$; Prader-Willi syndrome $(n=1)$; Sotos syndrome $(n=1)$; and intellectual disability subsequent to Reye's syndrome $(n=1)$.

Overall, $3.7 \%$ of the syndrome group were taking antipsychotic medication, $11.1 \%$ were taking anti-epileptic 


\begin{tabular}{|c|c|c|}
\hline Variable & $\begin{array}{l}\text { Cornelia de Lange } \\
\text { syndrome group }\end{array}$ & $\begin{array}{l}\text { Comparison } \\
\text { group }\end{array}$ \\
\hline \multicolumn{3}{|l|}{ Age, years } \\
\hline Mean (s.d.) & $13.88(8.58)$ & $13.74(7.99)$ \\
\hline Range & $3.18-37.91$ & $4.25-37.91$ \\
\hline \multicolumn{3}{|l|}{ Gender, \% } \\
\hline Male & 46 & 50 \\
\hline \multicolumn{3}{|c|}{ Wessex Scale (self-help), \% } \\
\hline Not able & 46 & 42 \\
\hline Partly able & 41 & 49 \\
\hline Able & 13 & 9 \\
\hline \multicolumn{3}{|c|}{ Wheelchair use, \% } \\
\hline Always & 11 & 11 \\
\hline Occasionally & 33 & 28 \\
\hline Never & 56 & 61 \\
\hline
\end{tabular}

medication and $42.6 \%$ other types of non-psychoactive medication. Of the comparison group, $10.9 \%$ of participants were on antipsychotic medication, $23.9 \%$ were taking anti-epileptic medication and $31.3 \%$ other forms of non-psychoactive medication.

\section{Measures}

Wessex scale

The Wessex Scale ${ }^{15}$ is an informant questionnaire designed to assess social and physical abilities in children and adults with intellectual disabilities. It has good interrater reliability at subscale level for both children and adults. ${ }^{15,16}$

\section{Vineland Adaptive Behavior Scales}

The Vineland Adaptive Behavior Scales (Interview Edition) ${ }^{17}$ measure ability in individuals from birth to 18 years 11 months and in 'low-functioning' adults. Three domains of adaptive behaviour were used: Communication, with sub-domains Receptive, Expressive and Written; Daily Living Skills; and Socialisation. Results were obtained as age-equivalent scores. Interrater and test-retest reliability indices, construct, content and criterion validity are robust. ${ }^{17}$

\section{Aberrant Behavior Checklist}

The Aberrant Behavior Checklist ${ }^{18}$ is a measure of problem behaviour incorporating a 58-item checklist with five factors based on observations of behaviour over the previous month, including: irritability, agitation and crying; lethargy and social withdrawal; stereotypic behaviour; inappropriate speech; and hyperactivity and non-adherence. The checklist is completed by caregivers, who rate each behaviour from 0 (not at all a problem) to 4 (problem is severe in degree). Aman \& $\operatorname{Singh}^{18}$ report robust interrater and test-retest reliability.

\section{Compulsive Behavior Checklist}

The Compulsive Behavior Checklist ${ }^{19}$ is a questionnaire measure comprising 25 items grouped into five categories: Ordering, Completeness, Cleaning, Checking and Grooming. The mean interrater reliability agreement across raters is $84.3 \% .^{20}$

\section{Childhood Autism Rating Scale}

The Childhood Autism Rating Scale ${ }^{21}$ is an observational rating scale incorporating 15 items developed to identify individuals with autism. Informants rate each behaviour from 1 (no evidence of autistic-type behaviour) to 4 (severe autistic-type behaviour). Aggregate scores of 15-29.5 indicate no autism, 30-36.5 mild to moderate autism and scores of over 37, severe autism. Scores are standardised based on normative data collected from 1500 individuals with autism. Interrater and test-retest reliability, criterion validity and internal consistency are reported to be good. $^{21}$

\section{Procedure}

Questionnaire and interview measures were completed by the participant's daytime carer. Observers visited each participant and collected videotaped observations of the participant for about $4 \mathrm{~h}$. Following data collection, the videotapes were used to rate relevant items on the Childhood Autism Rating Scale. Ethical review of all aspects of the study was obtained.

\section{Data analysis}

For the majority of analyses chi-squared or $t$-tests were used to compare groups. For analyses for which no prediction was made and multiple comparisons were undertaken (analyses of sub-scales for the Aberrant Behavior Checklist and the Compulsive Behavior Checklist), Bonferroni corrections were applied $(P<0.01)$.

When examining the behavioural phenotype across behaviours to control for covariance, a regression analysis was conducted to predict group membership. The following behavioural variables were force entered into a binary logistic regression equation: total number of compulsions as measured by the Compulsive Behavior Checklist, the total Childhood Autism Rating Scale score, total score on each factor of the Aberrant Behavior Checklist and Expressive Communication sub-domain age-equivalent scores. Post hoc tests (variance inflation factor) were applied to the data to test for multicollinearity.

\section{Results}

To ensure that the syndrome group did not differ from the comparison group on matching criteria, statistical comparisons were undertaken. The $t$-test and chi-squared statistics showed no significant difference between the two groups for age $(t(97)=-0.08, P=0.94)$, gender $\left(\chi^{2}(1)=0.14, P=0.71\right)$, ability $\left(\chi^{2}(1)=0.67, P=0.72\right)$ and mobility $\left(\chi^{2}(1)=0.33, P=0.85\right)$. Analysis of the Vineland Adaptive Behavior Scale data showed no difference between groups for mental-age-equivalent scores (syndrome group: mean=27.43 months (s.d.=20.67); comparison group: mean $=27.80$ months (s.d.=22.79); $t(97)=0.09, P=0.93)$. The percentage of participants with Cornelia de Lange syndrome with given degrees of intellectual disability were: profound $50.00 \%$, severe $24.07 \%$, moderate $14.81 \%$ and mild $11.11 \%$. Equivalent data for the comparison group were $45.65 \%, 30.43 \%, 15.22 \%$ and $8.70 \%$ respectively. These proportions did not differ significantly $\left(\chi^{2}(1)=0.62, P=0.89\right)$. In combination, these analyses showed that the groups were comparable.

To examine the potential discrepancy between receptive and expressive language abilities, mean age-equivalent scores for the Communication sub-domains of Receptive, Expressive and Written were compared across groups (Fig. 1). A mixed two-way ANOVA revealed an interaction effect $(F(2,196)=3.39, P<0.05)$. Examination of the data in Fig. 1 showed that the syndrome group performed relatively more poorly on the Expressive communication domain compared with other domains than the comparison group. 


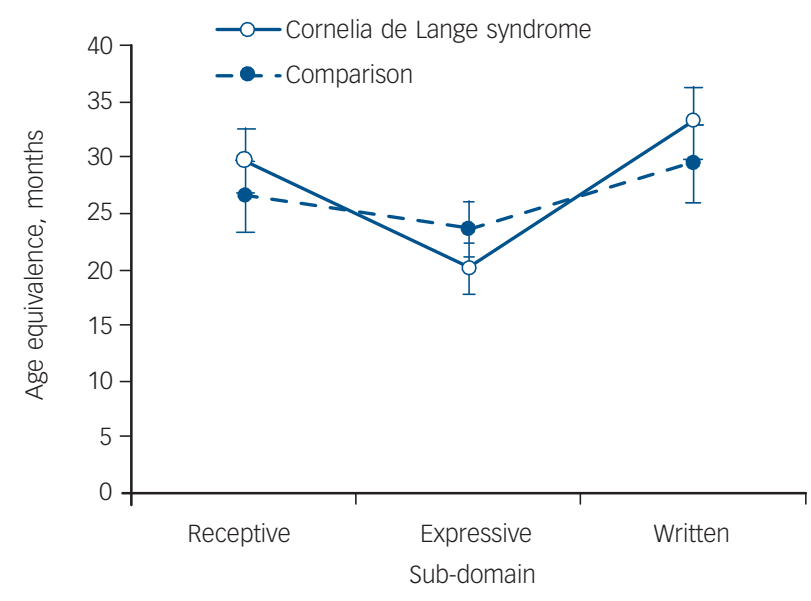

Fig. 1 Mean-age equivalent scores (in months) ( \pm 1 s.e.) for Communication sub-domain of the Vineland Adaptive Behavior Scales for both groups.

Table 2 shows the mean scores for the syndrome and comparison groups on the sub-scales of the Aberrant Behavior Checklist and the Compulsive Behavior Checklist. For the Aberrant Behavior Checklist there were no significant differences between the groups on sub-domains or total score (syndrome group: mean $=40.77$ (s.d. $=34.15$ ); comparison group: mean $=32.98$ (s.d.=30.87); $t(95)=1.71, P=0.24)$. On the sub-domains of the Compulsive Behavior Checklist, the syndrome group scored significantly higher on the Cleaning and Checking sub-scales ( $P=0.002$ and $P=0.006$ respectively) with the difference on the Grooming sub-scale approaching significance $(P=0.049)$. The syndrome group showed a significantly higher number of compulsive behaviours than the comparison group (mean $=4.12$ (s.d. $=3.99$ ) and mean $=2.67$ (s.d. $=3.16$ ) respectively; $t(95)=2.68, P=0.009$ ). Finally, $86.5 \%$ of participants with Cornelia de Lange syndrome and $57.8 \%$ of participants in the comparison group displayed one or more forms of compulsive behaviour $\left(\chi^{2}(1)=10.17, P<0.01\right)$.
Table 3 displays the percentage of participants in each category of autism. A significantly greater proportion of individuals in the syndrome group (32.1\%) scored in the 'severe autism' category compared with the comparison group $(7.1 \%)$.

\section{Predicting group membership}

A binary logistic regression was conducted to determine the behavioural characteristics that predicted group membership, while controlling for other behaviours. The regression equation was conducted with the following variables: total number of compulsions as measured by the Compulsive Behavior Checklist, the Childhood Autism Rating Scale score, total score on each factor of the Aberrant Behavior Checklist, and Expressive Communication age-equivalent scores from the Vineland Adaptive Behavior Scale. The regression model correctly classified $64 \%$ of cases. Total score on the Childhood Autism Rating Scale (Wald=8.34, d.f.(1), $P<0.01, O R=1.15$ ) and total number of compulsions (Wald=4.44, d.f.(1), $P<0.05, \mathrm{OR}=1.23$ ) significantly predicted group membership when all other variables were controlled for. Post hoc tests revealed that the data did not show multicollinearity.

To examine compulsive behaviours in the syndrome and comparison groups in more detail, a binary logistic regression was also carried out with the five Compulsive Behavior Checklist categories as independent variables. The regression model correctly classified $66 \%$ of cases, with the number of cleaning compulsions being the only significant variable predicting group membership (Wald=7.74, d.f.(1), $P<0.01, \mathrm{OR}=2.36$ ). Post hoc tests revealed that the data did not show multicollinearity.

\section{Discussion}

\section{Intellectual disability and impairment of communication}

The majority $(76 \%)$ of participants with Cornelia de Lange syndrome were classified as having severe and profound intellectual disability and this distribution of degree of intellectual

\begin{tabular}{|c|c|c|c|c|}
\hline Measure & $\begin{array}{l}\text { Cornelia de Lange syndrome group } \\
\text { Mean (s.d.) }\end{array}$ & $\begin{array}{l}\text { Comparison group } \\
\text { Mean (s.d.) }\end{array}$ & $t$ & $P$ \\
\hline \multicolumn{5}{|c|}{ Aberrant Behavior Checklist } \\
\hline Irritability & $10.63(9.37)$ & $7.98 \quad(8.76)$ & 1.17 & 0.24 \\
\hline Lethargy & $9.12(10.07)$ & $8.31(10.16)$ & 1.42 & 0.16 \\
\hline Stereotypy & $4.23 \quad(5.27)$ & $3.20 \quad(4.43)$ & 0.94 & 0.35 \\
\hline Hyperactivity & $13.02(12.68)$ & $11.10(11.64)$ & 0.78 & 0.44 \\
\hline Inappropriate speech & $1.59(2.77)$ & $1.02(2.32)$ & 1.10 & 0.28 \\
\hline \multicolumn{5}{|c|}{ Compulsive Behaviour Checklist } \\
\hline Ordering & $1.15(1.58)$ & $0.88 \quad(1.20)$ & 1.09 & 0.28 \\
\hline Completeness & $0.79(1.23)$ & $0.44 \quad(0.14)$ & 1.61 & 0.11 \\
\hline Cleaning & $0.85(1.02)$ & $0.29 \quad(0.66)$ & 3.24 & 0.002 \\
\hline Checking & $0.81(0.58)$ & $0.33 \quad(0.64)$ & 2.80 & 0.006 \\
\hline Grooming & $0.58(0.64)$ & $0.33 \quad(0.56)$ & 2.00 & 0.049 \\
\hline
\end{tabular}

Table 3 Percentage of participants in each category of autism (as defined by the Childhood Autism Rating Scale) broken down by group

\begin{tabular}{|lcccc|}
\hline Group & Non-autistic & Mild to moderate autism & Severe autism & $\chi^{2}$ \\
\hline Cornelia de Lange syndrome group & 52.8 & 15.1 & 32.1 & 8.77 \\
\hline Comparison group & 71.4 & 21.4 & 0.012 & 7.1 \\
\hline
\end{tabular}




\begin{tabular}{|c|c|c|c|c|c|c|c|}
\hline \multirow[b]{2}{*}{ Study } & \multirow[b]{2}{*}{ Measure } & \multirow[b]{2}{*}{$n$} & \multicolumn{5}{|c|}{ Participants scoring within categories of intellectual disability, \% } \\
\hline & & & Profound & Severe & Moderate & Mild & Borderline /normal \\
\hline This study & Vineland Adaptive Behavior Scale & 54 & 50 & 24 & 15 & 11 & 0 \\
\hline Beck $(1987)^{12}$ & Vineland Social Maturity Scale & 36 & 50 & 14 & 17 & 6 & 14 \\
\hline Berney et al (1999) ${ }^{6}$ & Not reported & 49 & 43 & 20 & 18 & 8 & 10 \\
\hline
\end{tabular}

disability is similar to that previously reported (Table 4). ${ }^{6,12}$ This confirms the broad range of intellectual disability in the syndrome and the data in Table 4 suggest that the current sample is representative. Previous literature has indicated that individuals with this syndrome may have deficits in expressive communication relative to their overall adaptive behaviour. ${ }^{7,12}$ In this study we identify a deficit of expressive communication in Cornelia de Lange syndrome relative to receptive and written communication that appears to be phenotypic of the syndrome. Future research should employ more sophisticated assessments of language in individuals with the syndrome to investigate this relative deficit as the Vineland Adaptive Behavior Scale is a crude assessment of communication. Additionally, further research should examine the potential role of hearing impairment as contributory to this profile.

\section{Autistic-spectrum disorder and compulsive behaviour}

Previous studies have suggested that individuals with Cornelia de Lange syndrome may have an increased likelihood of showing autistic-type behaviour. ${ }^{6,23}$ Our study supports this assertion as individuals with the syndrome were significantly more likely to be categorised as 'severely autistic' than individuals in the comparison group. Furthermore, results of a regression analysis to examine the contribution of characteristics to the prediction of group membership indicated that total score on the Childhood Autism Rating Scale was a significant predictor. Thus, variables such as degree of intellectual disability that are known to influence prevalence of autism ${ }^{24}$ have been controlled for both in this analysis and in the experimental design. Although this finding is important it requires replication using psychometrically robust measures of autism combined with developmental history and assessments that inform established diagnostic algorithms. Comparisons of phenomenology of autism not associated with syndromes and in high-risk syndromes, such as fragile- $\mathrm{X}$ and tuberous sclerosis, are also warranted.

It is notable that no differences were found between the groups on the Aberrant Behavior Checklist at the full-scale or sub-scale level. Previous research has indicated that individuals with Cornelia de Lange syndrome may have an increased likelihood of showing hyperactive and stereotyped behaviour. ${ }^{6}$ However, our study demonstrates that when employing a matched comparison group no differences are found. The finding of no difference in global behaviour disorder between the two groups also indicates the need for the use of specific measures of behaviour in this type of research given other identified differences.

This study provides further evidence of a high prevalence of compulsive behaviour in Cornelia de Lange syndrome. Consistent with the report of Hyman et al, ${ }^{14}$ over $85 \%$ of participants in the syndrome group showed compulsive behaviour, significantly more than in the comparison group. Analyses also indicated that the syndrome group displayed more compulsions than the comparison group and, critically, the regression analysis demonstrated that the number of compulsions was a reliable predictor of Cornelia de Lange syndrome when autism was controlled for. Although this finding is robust there is need for caution regarding significance. It is not clear that the Compulsive Behavior Checklist is assessing compulsive behaviour as defined by DSM-IV ${ }^{25}$, for example, and some stereotyped behaviours might be included in the assessment. Further research should clarify the phenomenology of the compulsive behaviours and undertake detailed comparisons with other groups.

\section{Future research}

Our findings are important for a number of reasons. First, differences between the groups were found for an expressive communication deficit, compulsive behaviours and autism, and these characteristics appear to be phenotypic of Cornelia de Lange syndrome. Second, the results indicate that compulsive behaviours might be more pronounced in this syndrome than would be predicted from the presence of other autistic impairments and this dissociation alludes to the potential for an additional pathway to aspects of the behavioural phenotype. Future research should focus on further delineation of the phenomenology of the phenotypic characteristics noted in this study and their relationship with the genetic subtypes of Cornelia de Lange syndrome that are now being described. ${ }^{13}$

Chris Oliver, BSC, MPhil, PhD, Kate Arron, BSC, MPhil, ClinPsyD, Jenny Sloneem BSC, PhD, DClinPsy, School of Psychology, University of Birmingham, UK; Scott Hall, PhD, Department of Psychiatry and Behavioral Science, Stanford University, USA

Correspondence: Chris Oliver, Centre for Neurodevelopmental Disorders, School of Psychology, University of Birmingham, Edgbaston, Birmingham B15 2TT, UK. Email: c.oliver@bham.ac.uk

First received 24 Aug 2007, final revision May 2008, accepted 12 Jun 2008

\section{Acknowledgements}

This research was supported by a grant from the PPP Foundation. J.S. was supported by a PhD studentship from the Medical Research Council. We are grateful to the Cornelia de Lange Syndrome Foundation (UK and Ireland) for their support, the families and carers of participants and the participants themselves for their diligence and patience. We are grateful to Dr Tonie Kline for her contribution to the diagnostic procedure.

\section{References}

1 Krantz ID, McCallum J, Descipio C, Kaur M, Gillis LA, Yaeger D, Jukofsky L, Wasserman N, Bottani A, Morris CA, Nowaczyk MJM, Toriello H, Bamshad M.J, Carey JC, Rappaport E, Kawauchi S, Lander AD, Calof AL, Li H, Devoto M, Jackson LG. Cornelia de Lange syndrome is caused by mutations in NIPBL, the human homolog of Drosophila melanogaster Nipped-B. Nat Genet 2004; 36: 631-5.

2 Tonkin E, Wang TJ, Lisgo S, Bamshad MJ, Strachan T. NIPBL, encoding a homolog of fungal Scc2-type sister chromatid cohesion proteisn and fly Nipped-B, is mutated in Cornelia de Lange syndrome. Nat Genet 2004; 36 : 636-41.

3 Musio A, Selicorni A, Focarelli ML, Gervasini C, Milani D, Russo S, Vezzoni $P$, Larizza L. X-linked Cornelia de Lange syndrome owing to SMC1L1 mutations. Nat Genet 2006; 38: 528-30.

4 Deardorff MA, Kaur M, Yaeger D, Rampuria A, Korolev S, Pie J, Gil-Rodríguez C, Arnedo M, Loeys B, Kline AD, Wilson M, Lillquist $K$, Siu V, Ramos FJ, Musio A, Jackson LS, Dorsett $D$, Krantz ID. Mutations in cohesin complex members SMC3 and SMC1A cause a mild variant of Cornelia de Lange syndrome with predominant mental retardation. Am J Hum Genet, 2007; 80: 485-94. 
5 Kline AD, Krantz ID, Sommer A, Kleiwer M, Jackson LD, Fitzpatrick DR, Levin AV, Selicorni A. Cornelia de Lange syndrome: clinical review, diagnostic and scoring systems, and anticipatory guidance. Am J Med Genet A 2007; 143A 1287-96.

6 Berney TP, Ireland M, Burn J. Behavioural phenotype of Cornelia de Lange syndrome. Arch Dis Child 1999; 81: 333-6.

7 Kline AD, Stanley C, Belevich J, Brodsky K, Barr M, Jackson LG. Developmental-data on individuals with the Brachmann-delange syndrome. Am J Med Genet 1993; 47: 1053-8.

8 Hawley PP, Jackson LG, Kurnit DM.. 64 patients with Brachmann-de-Lange syndrome - a survey. Am J Med Genet 1985; 20: 453-9.

9 Arron K, Oliver C, Hall S, Sloneem J, Forman D, McClintock K. Effects of Socia Interaction on Pragmatic Communication and Self-Injurious Behavior in Cornelia de Lange syndrome. Am J Ment Retard 2006; 111: 184-92.

10 Moss J, Oliver C, Hall S, Arron K, Sloneem J, Petty J. The association between environmental events and self-injurious behaviour in Cornelia de Lange syndrome. J Intellect Disabil Res 2005; 49: 269-77.

11 Basile E, Villa L, Selicorni A, Molteni M. The behavioural phenotype of Cornelia de Lange Syndrome: a study of 56 individuals. J Intellect Disabil Res 2007; 51: 671-81.

12 Beck B. (1987) Psycho-social assessment of 36 de Lange patients. J Ment Defic Res 1987; 31: 251-7.

13 Bhuiyan Z, Klien M, Hammond P, Mannens M, Van Haeringen A, Van Berckelaer-Onnes I, Hennekam RC. Genotype-Phenotype correlations of 39 patients with Cornelia de Lange syndrome: the Dutch experience. J Med Genet 2006; 43: 568-75

14 Hyman P, Oliver C, Hall S. (2002). Self-injurious behavior, self-restraint, and compulsive Behaviors in Cornelia de Lange syndrome. Am J Ment Retard 2002; 107: 146-54.
15 Kushlick A, Blunden R, Cox,G. A method of rating behaviour characteristics for use in large scale surveys of mental handicap. Psychol Med 1973; 3 : $466-78$.

16 Palmer J, Jenkins J. The 'Wessex' behaviour rating system for mentally handicapped people: reliability study. Br J Ment Subnorm 1982; 28: 88-96.

17 Sparrow SS, Balla DA, Cicchetti DV. The Vineland Adaptive Behavior Scales: Interview Edition. American Guidance Service, 1984.

18 Aman MG, Singh NN. The Aberrant Behavior Checklist: Manual. Slosson Educational Publications, 1986

19 Gedye,A. Recognizing obsessive compulsive disorder in clients with developmental disabilities. Habilitative Mental Healthcare Newsletter, 1992 11: 73-7.

20 Bodfish JW, Crawford TW, Powell SB, Parker DE, Golden RN, Lewis MH. Compulsions in adults with mental-retardation - prevalence, phenomenology, and comorbidity with stereotypy and self-injury. Am J Ment Retard 1995; 100: 183-92.

21 Schopler E, Reichler R J, Renner BR. The Childhood Autism Rating Scale (CARS). Irvington Publishers, 1988

22 Sturmey P, Matson J, Sevin JA. Brief report: Analysis of the internal consistency of three autism scales. J Autism Dev Disord 1992; 22: 321-8.

23 Moss J, Oliver C, Berg K, Kaur G, Jephcott L, Cornish K. The prevalence and phenomenology of autistic spectrum disorder in Cornelia de Lange and Cri du Chat syndromes. Am J Ment Retard 2008; 113: 278-91.

24 Nordin V, Gillberg C. Autism spectrum disorders in children with physical or mental disability or both. II: Screening aspects. Dev Med Child Neurol 1996; 38: $314-24$.

25 American Psychiatric Association. Diagnostic and Statistical Manual of Mental Disorders (4th edn) (DSM-IV). APA, 1994. 